\title{
Atividade de Ondas de Gravidade na Estratosfera Terrestre Inferida Através da Técnica de Rádio Ocultação de GPS
}

\author{
Cristiano Max Wrasse ${ }^{1}$, Hisao Takahashi ${ }^{1}$, Joaquim Fechine1, Jens Wickert ${ }^{2}$, Clezio De Nardin ${ }^{1}$ \\ ${ }_{1}^{1}$ Instituto Nacional de Pesquisas Espaciais - INPE - São José dos Campos (SP) \\ ${ }^{2}$ GeoForschungsZentrum - Potsdam - Alemanha
}

Copyright 2005, SBGf - Sociedade Brasileira de Geofísica

This paper was prepared for presentation at the $9^{\text {th }}$ International Congress of the Brazilian Geophysical Society held in Salvador, Brazil, 11-14 September 2005.

Contents of this paper were reviewed by the Technical Committee of the $9^{\text {th }}$ International Congress of the Brazilian Geophysical Society. Ideas and concepts of the text are authors' responsibility and do not necessarily represent any position of the SBGf, its officers or members. Electronic reproduction or storage of any part of this paper for commercial purposes without the written consent of the Brazilian Geophysical Society is prohibited.

\begin{abstract}
Neste trabalho são apresentados alguns resultados da atividade de ondas de gravidade na estratosfera sobre a América Latina, inferida através da técnica de rádio ocultação de GPS. Os dados utilizados foram obtidos pelo satélite Challenging Minisatellite Payload (GPSCHAMP). Também serão apresentados, a teoria e a metodologia empregada no cálculo da atividade das ondas de gravidade.
\end{abstract}

\section{Introdução}

Sabe-se que as ondas de gravidade exercem a maior influência em larga escala na circulação e na estrutura da atmosfera. As ondas de gravidade são consideradas as responsáveis por muitas das variações espaciais (da ordem de alguns quilômetros a dezenas de quilômetros) e temporais (de $\sim 5$ min à várias horas) em muitas das variáveis atmosféricas acima da tropopausa. As fontes de geração destas ondas de gravidade incluem fenômenos meteorológicos como as frentes frias, os sistemas convectivos, vento soprando sobre montanhas entre outros. A maioria destas fontes de geração encontra-se na troposfera (Fritts e Nastrom, 1992). Nos trópicos, a geração das ondas de gravidade está associada à convecção, gerando grandes nuvens do tipo cumulus nimbus.

As ondas de gravidade também são responsáveis pelo transporte de energia e momentum entre a baixa e a média atmosfera sendo uma das responsáveis pelo acoplamento dinâmico entre as camadas da atmosfera. Durante as ultimas décadas, radares de VHF (Very High Frequency), de Laser, entre outros instrumentos, tem sido utilizados para caracterizar as ondas de gravidade. Apesar destes instrumentos apresentam boa resolução temporal e espacial a rede destes equipamentos de solo encontram-se muito dispersa para se ter uma cobertura global. Desta forma, observações realizadas por satélites são capazes de produzir uma grande cobertura global (Wu e Waters, 1996), porém não apresentam uma boa resolução espacial. Entretanto, estas medidas podem fornecer uma quantitativa distribuição da atividade das ondas de gravidade. Recentemente, Tsuda et all. (2000) utilizou dados do satélite GPS-MET para realizar uma análise global da atividade de ondas de gravidade na estratosfera. Assim, no presente trabalho serão apresentados, a teoria, a metodologia e alguns resultados da atividade de onda de gravidade obtidos a partir dos dados do satélite GPS-CHAMP para a América Latina.

\section{Teoria do Calculo da Densidade de Energia das Ondas de Gravidade}

Flutuações de mesoescala observadas na velocidade do vento e na temperatura aparecem como uma superposição de varias ondas de gravidade com diferentes escalas espacial e temporal. Desta forma, o comportamento das ondas de gravidade é muitas vezes melhor interpretado em termos do espectro como uma função da freqüência da onda $\omega$ e dos números de onda vertical e horizontal $m$ e $k$. VanZandt (1982) investigou $o$ espectro das ondas de gravidade observadas na troposfera e na media atmosfera e encontrou uma universalidade do espectro em relação as estações do ano, altitude e condições geográficas. Em particular, a freqüência e o numero de onda horizontal do espectro das ondas de gravidade variam, $\omega^{-5 / 3}$ e $k^{-5 / 3}$, respectivamente.

Utilizando perfis da velocidade do vento e da temperatura observados por radares e por radiossondas Fritts e VanZandt (1993) descrevem a energia do espectro para as perturbações na velocidade do vento e na temperatura causadas pelas ondas de gravidade, como um produto de funções separadas em $\omega, m$ e $\phi$ dada pela seguinte expressão:

$$
E(\mu, \omega, \phi)=E_{0} A(\mu) B(\omega) \Phi(\phi)
$$

Com,

$$
\begin{gathered}
A(\mu)=\frac{A_{0} \mu^{s}}{\left(1+\mu^{s+t}\right)} \\
B(\omega)=B_{0} \omega^{-p}
\end{gathered}
$$

Onde, $\mu=m / m_{*}$ e $m=2 \pi / \lambda_{z} . \quad \lambda_{z}$ e $m_{*}$ são respectivamente, o comprimento de onda vertical e o numero de onda característico e $\phi$ é o angulo azimutal indicando a direção de propagação da onda. O parâmetro 
$E_{0}$ representa a energia total das ondas de gravidade por unidade de massa, enquanto $A_{0}$ e $B_{0}$ são os parâmetros normalizados para o numero de onda vertical e para o espectro de freqüência, $A(\mu)$ e $B(\omega)$. A função $\Phi(\phi)$ representa a anisotropia das ondas de gravidade.

A densidade de energia $E_{0}$, que pode ser utilizada para medir a atividade de ondas de gravidade, é definida por (Allen and Vincent, 1995; Tsuda et al., 2000):

$$
E_{0}=E_{k}+E_{p}=\frac{1}{2}\left[\overline{u^{\prime 2}}+\overline{v^{\prime 2}}+\overline{w^{\prime 2}}+\left(\frac{g}{N}\right)^{2} \overline{\left(\frac{T^{\prime}}{\bar{T}}\right)^{2}}\right.
$$

Onde $E_{k}$ e $E_{p}$ representam as energias cinética e potencial por unidade de massa, respectivamente. Estas energias podem ser reescritas da seguinte forma:

$$
\begin{array}{r}
E_{k}=\frac{1}{2}\left[\overline{u^{\prime 2}}+\overline{v^{\prime 2}}+\overline{w^{\prime 2}}\right] \\
E_{p}=\frac{1}{2}\left(\frac{g}{N}\right)^{2} \overline{\left(\frac{T}{\bar{T}}\right)^{2}}
\end{array}
$$

$\mathrm{E}$

$$
N^{2}=\frac{g}{T}\left(\frac{d T}{d z}+\frac{g}{C_{p}}\right)
$$

Os termos $u^{\prime}, v^{\prime}$ e $w^{\prime}$ representam as componentes perturbadas da velocidade do vento, nas direções zonal, meridional e vertical, respectivamente; $g$ e a aceleração devido a gravidade; $N$ e a freqüência de Brunt-Väisälä; $\bar{T}$ e $T^{\prime}$ são as componentes media e perturbada da temperatura; $C_{p}$ é o calor especifico à pressão constante do ar seco. De acordo com a teoria linear das ondas e gravidade, a razão entre a energia cinética e potencial torna-se constante, portanto é possível estimar $E_{0}$ apenas a partir das observações da temperatura (Tsuda et al., 2000). Desta forma, o calculo da energia potencial depende principalmente da estimativa da flutuação da temperatura causada pelas ondas de gravidade.

\section{Metodologia para Determinar a Atividade de Ondas de Gravidade}

Para o calculo da energia potencial, $E_{p}$, é necessário conhecer, além do perfil de temperatura, o perfil da flutuação da temperatura, a variância da flutuação e a freqüência de Brunt-Väisälä.

O perfil da temperatura atmosférica, $T(z)$, pode ser considerado como sendo formado pela temperatura básica, $\bar{T}(z)$,e pela componente da flutuação, $T^{\prime}(z)$. A flutuação da temperatura é então obtida aplicando-se um filtro passa-alta com uma freqüência de corte de 10 $\mathrm{km}$. Procedimentos semelhantes foram realizados nas analises de perfis dos parâmetros atmosféricos obtidos por foguetes (e.g Eckermann et al., 1994). A freqüência de Brunt-Väisälä ao quadrado, $N^{2}$, também pode ser determinada através da diferenciação do perfil de temperatura em ralação a três alturas adjacentes.

A variância da flutuação da temperatura, $\overline{T^{\prime 2}}$, é determinada através da seguinte relação:

$$
\overline{T^{\prime 2}}=\frac{1}{z^{\max }-z^{\min }} \int_{z^{\min }}^{z^{\max }} T^{\prime 2} d z
$$

Onde, $z^{\max }$ e $z^{\text {min }}$ representam o topo e a base da região onde é realizado o calculo a energia potencial.

A Figura 1-A apresenta um exemplo de um perfil de temperatura obtido pelo satélite GPS-CHAMP na região nordeste do Brasil $\left(-4,05^{\circ} \mathrm{S},-34,05^{\circ} \mathrm{O}\right)$ no dia $17 / 08 / 2004$ em torno das 13:26 UT. Note que o perfil de temperatura foi filtrado com um filtro passa-baixa para a remoção do ruído. Na Figura 1-B é apresentada a flutuação da temperatura determinada através da filtragem do perfil de temperatura com um filtro-passa alta (com uma freqüência de corte de $10 \mathrm{~km}$ ). A Figura 1-C apresenta o perfil da freqüência de Brunt-Väisälä, $N^{2}$, ao quadrado, obtido a partir do perfil de temperatura (Figura 1-A). Como é de se esperar, o valor de $N^{2}$ é pequeno na troposfera e apresenta um aumento abrupto na altura da tropopausa, enquanto que valores maiores são observados na região da estratosfera ( 17 a $35 \mathrm{~km}$ de altura). Na Figura 1-D é apresentado um perfil da energia potencial, $E_{p}$, entre 20 e $35 \mathrm{~km}$ de altitude. Observa-se que os valores de $E_{p}$ apresentam os maiores valores entre 25 e $30 \mathrm{~km}$, variando entre $\sim 2$ e $6 \mathrm{~J} / \mathrm{kg}$.

\section{Resultados e Discussões}

Utilizando a metodologia descrita na seção anterior é possível calcular a distribuição da Energia Potencial $\left(E_{p}\right)$ para qualquer região de interesse. Devido à órbita polar realizada pelo satélite GPS-CHAMP os perfis de temperatura são obtidos globalmente.

A Figura 2-A apresenta o mapa de rádio ocultações ocorridas na América do Sul durante o outono de 2004 (entre fevereiro e maio de 2004). Cada ponto do mapa representa um perfil de temperatura obtido através da técnica de rádio ocultação. A Figura 2-B apresenta a distribuição da energia potencial, calculada para este período. Nota-se que as regiões com cores em verde e amarelo apresentam maiores valores de energia potencial e, por conseqüência, uma maior atividade de ondas de gravidade entre 20 e $30 \mathrm{~km}$ de altura. Observase também que a região de maior atividade de ondas de gravidade está distribuída $\pm 10^{\circ} \mathrm{em}$ torno do equador. Outras regiões isoladas com maior energia potencial podem ser notadas

A Figura 2-C apresenta a distribuição da temperatura na tropopausa. Observa-se que as menores temperaturas em torno de 180 a $190 \mathrm{k}$ na altura da tropopausa concentram-se em torno de $\pm 20^{\circ} \mathrm{em}$ torno do equador. 
Nota-se um aumentando desta temperatura, em torno de 200 a $230 \mathrm{~K}$, para latitudes maiores de $30^{\circ} \mathrm{S}$.

Por outro lado, a Figura 2-D apresenta a distribuição da altura da tropopausa para esta região. Observa-se que a altura maior da tropopausa está localizada em torno de \pm $30^{\circ} \mathrm{em}$ torno do equador, variando entre 14 e $19 \mathrm{~km}$ de altura. Nesta região nota-se que a temperatura é menor (Figura 2-C). Acima de $30^{\circ} \mathrm{S}$, observa-se que a altura da tropopausa varia entre 8 e $14 \mathrm{~km}$ de altura. Nesta mesma região observam-se temperaturas maiores para a região da tropopausa.

Os resultados da temperatura e da altura da tropopausa estão de acordo com as observações realizada por rádio sondagens que mostram uma altura da tropopausa maior no equador e menor à medida que se aproxima dos pólos. O inverso ocorre com a temperatura na altura da tropopausa, onde ocorre uma menor temperatura em torno do equador e maiores temperaturas próximas aos pólos.

\section{Sumário}

A partir dos perfis de temperatura obtidos pelo satélite GPS-CHAMP foi possível determinar a atividade de ondas de gravidade na região da estratosfera terrestre. Os resultados apresentados mostram que esta é uma técnica muito útil para o monitoramento global da atividade de ondas que gravidade na baixa atmosfera.

\section{Referências}

Allen, S. J.;Vincent, R. A. Gravity-Wave Activity in the Lower Atmosphere - Seasonal and Latitudinal Variations.
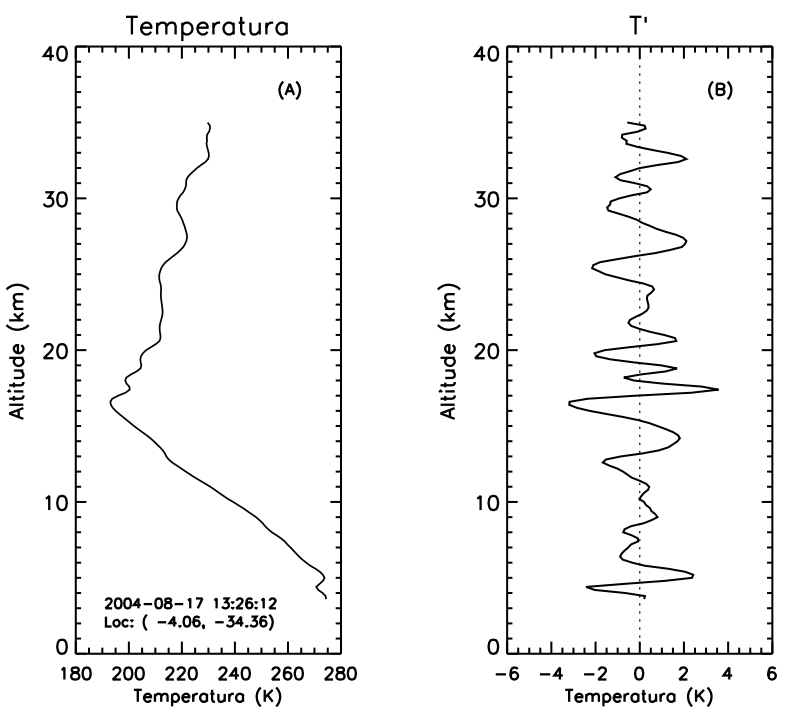

Journal of Geophysical Research-Atmospheres, v.100, n.D1, p. 1327-1350, JAN 201995.

Eckermann, S.D.; Hirota, I.; Hocking, W. K.; Gravity wave and equatorial wave morphology of the stratosphere derived from long-term rocket sounding. Q. J. R. Meteorol. Soc., 121, 149-186,1994.

Fritts, D. C.; Nastrom, G. D. Sources of Mesoscale Variability of Gravity-Waves .2. Frontal, Convective, and Jet-Stream Excitation. Journal of the Atmospheric Sciences, v.49, n.2, p. 111-127, JAN 151992.

Fritts, D. C.;Vanzandt, T. E. Effects of Doppler Shifting on the Frequency-Spectra of Atmospheric Gravity-Waves. Journal of Geophysical Research-Atmospheres, v.92, n.D8, p. 9723-9732, AUG 201987.

Tsuda, T.;Nishida, M.;Rocken, C.;Ware, R. H. A global morphology of gravity wave activity in the stratosphere revealed by the GPS occultation data (GPS/MET). Journal of Geophysical Research-Atmospheres, v.105, n.D6, p. 7257-7273, MAR 272000.

Vanzandt, T. E. A Universal Spectrum of Buoyancy Waves in the Atmosphere. Geophysical Research Letters, v.9, n.5, p. 575-578, 1982.

Wu, D. L.;Waters, J. W. Satellite observations of atmospheric variances: A possible indication of gravity waves. Geophys. Res. Lett., v.23, n.24, p. 3631-3634, 1996.
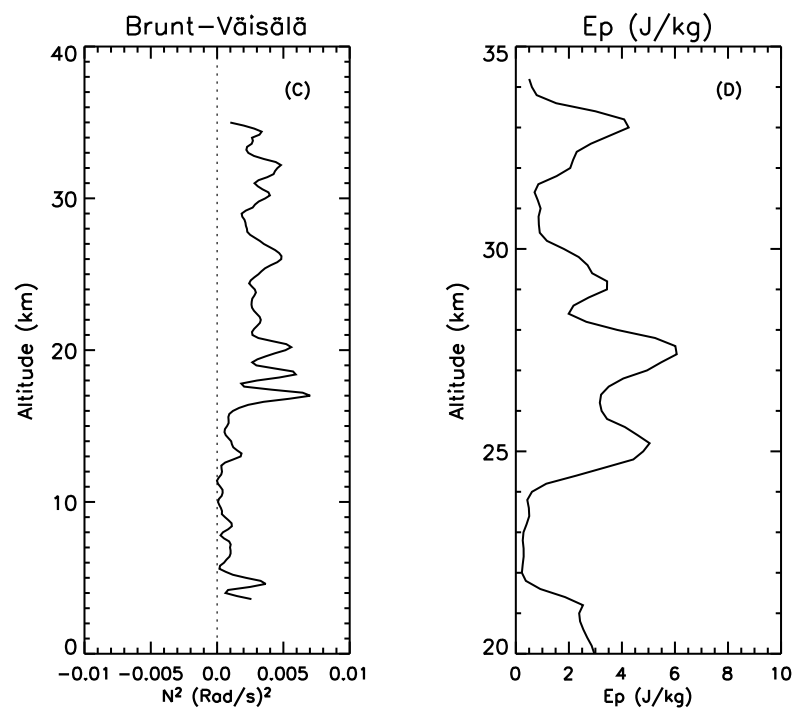

Figura 1 - (A) Perfil vertical de temperatura observado no dia 17/08/2004 na região nordeste do Brasil $\left(-4,05^{\circ} \mathrm{S},-34,05^{\circ} \mathrm{O}\right)$ em torno das 13:26 UT. (B) Perturbação da temperatura. (C) Freqüência de Brunt-Väisälä ao quadrado e (D) perfil de energia potencial $E_{p}$. 
(A) Radio Occultations 04/02/2004-05/05/2004

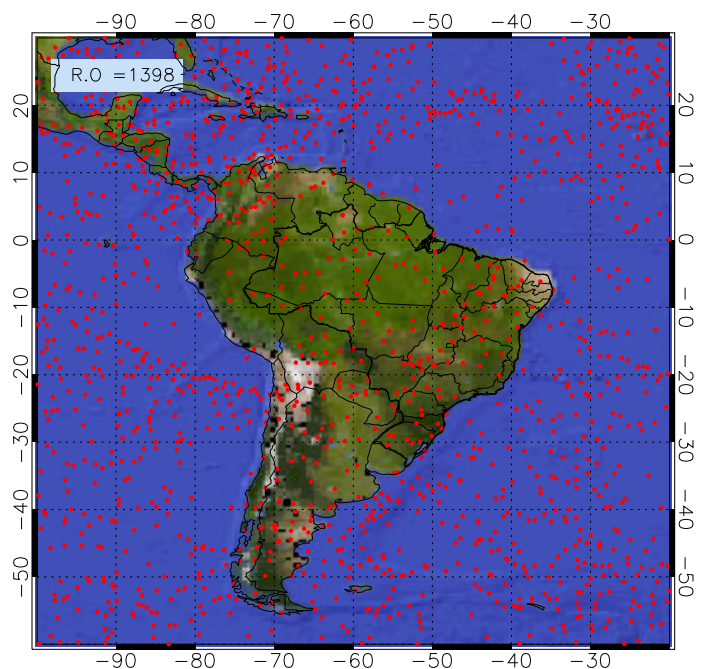

(C) Tropopouse Temperature (K) 04/02/2004 - 05/05/2004

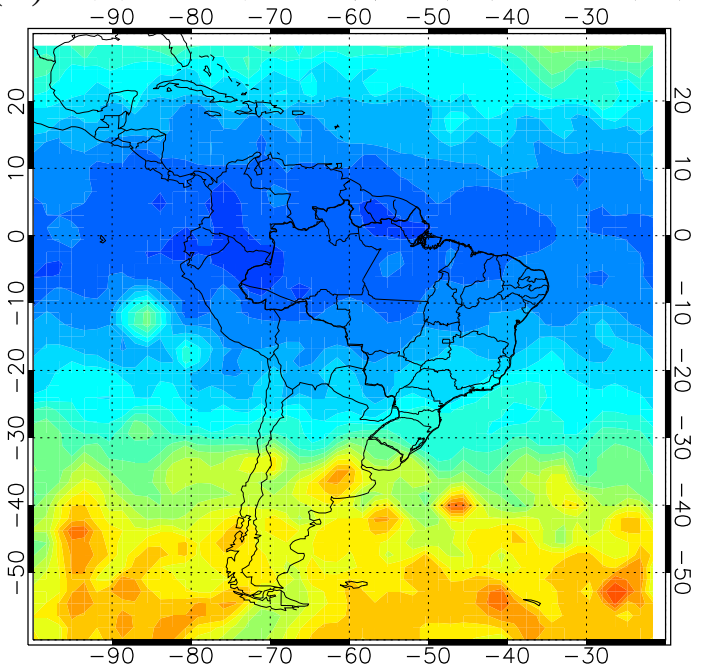

(B) $\mathrm{Ep}(\mathrm{J} / \mathrm{kg})(20-30 \mathrm{~km}) \quad 04 / 02 / 2004-05 / 05 / 2004$

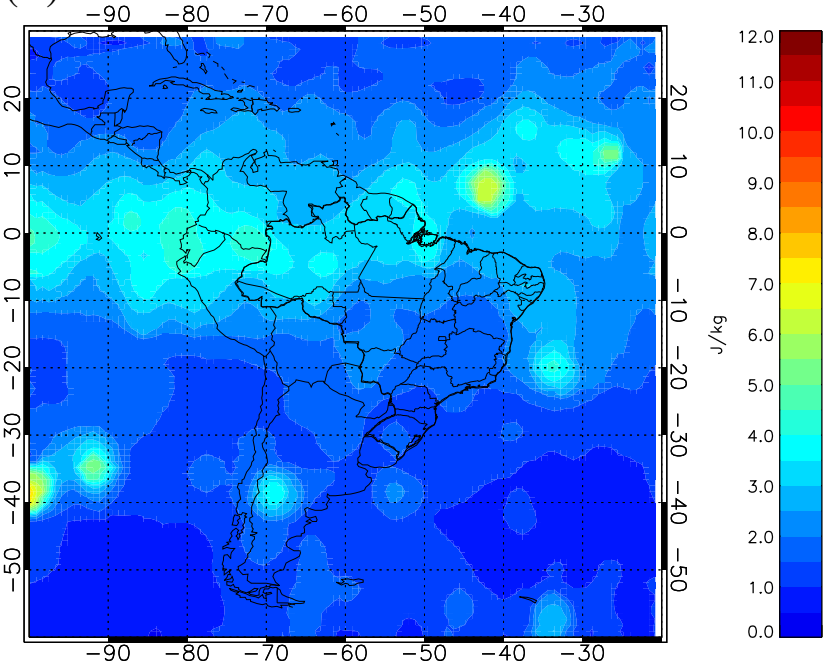

(D) Tropopouse Height (km) 04/02/2004 - 05/05/2004

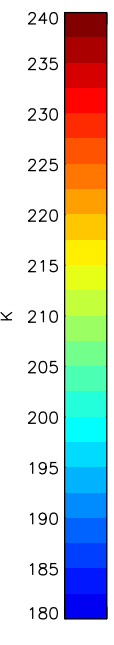

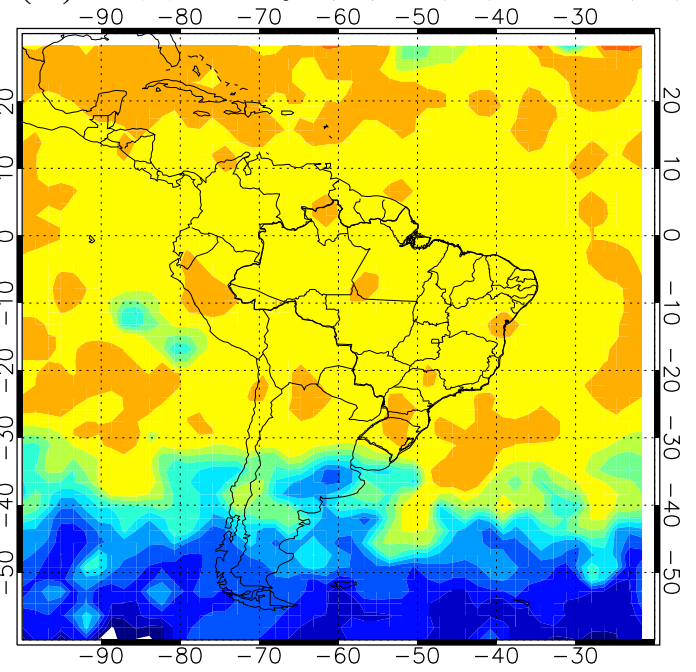

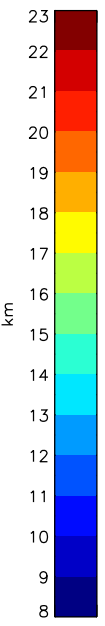

Figura 2 - (A) Número de rádio ocultações ocorridas durante o outono de 2004. (B) Energia potencial entre 20 e $30 \mathrm{~km}$ de altura. (C) Temperatura na Tropopausa. (D) Altura da Tropopausa. 

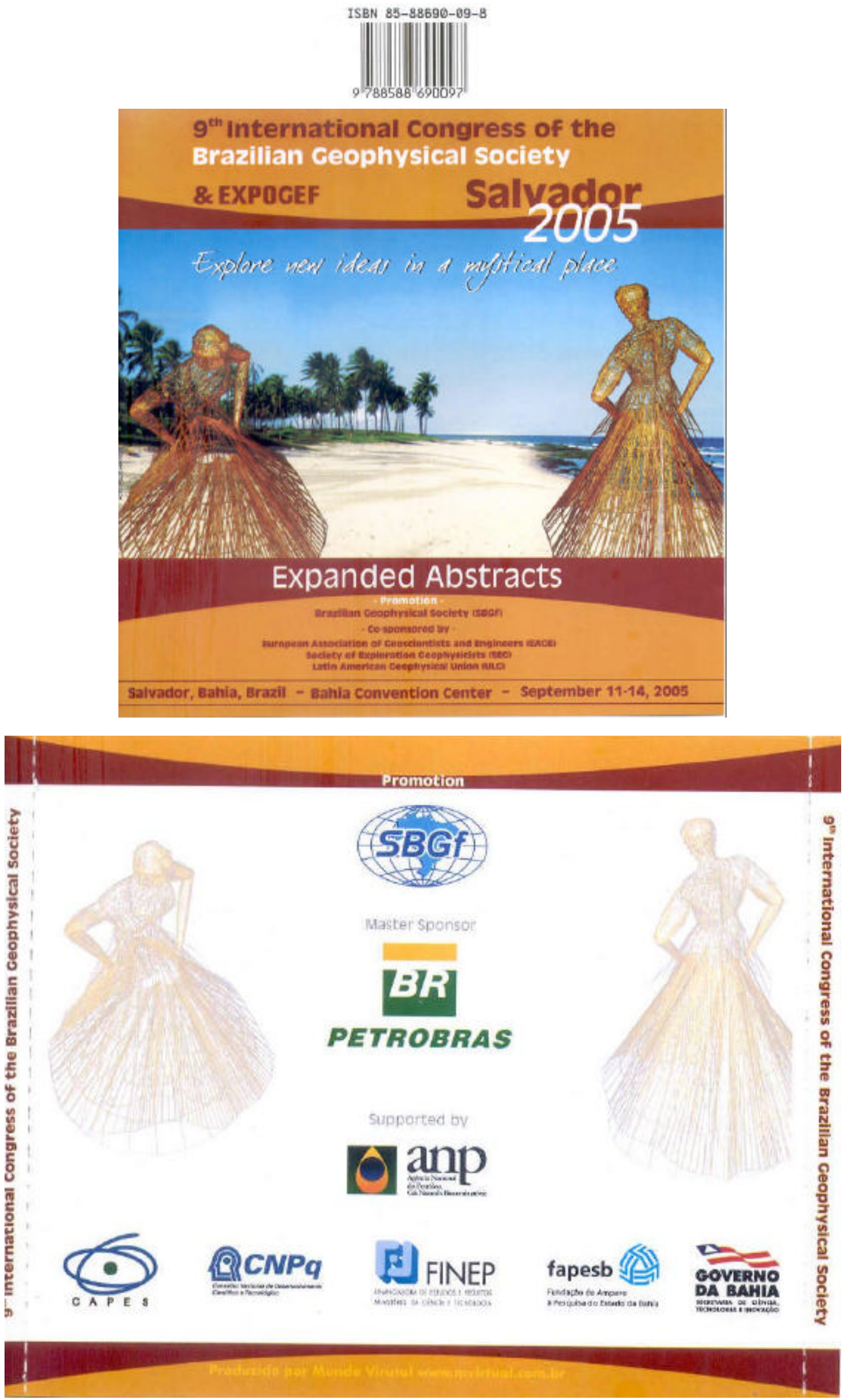